\title{
Calibration Proposal for New Antenna Array Architectures and Technologies for Space Communications
}

\author{
Miguel Alejandro Salas Natera. \\ Leandro de Haro Ariet,
}

\author{
Ramón Martínez Rodríguez-Osorio, \\ and Manuel Sierra Pérez.
}

\begin{abstract}
In large antenna arrays with a large number of antenna elements, the required number of measurements for the characterization of the antenna array is very demanding in cost and time. This letter presents a new offline calibration process for active antenna arrays that reduces the number of measurements by subarray-level characterization. This letter embraces measurements, characterization, and calibration as a global procedure assessing about the most adequate calibration technique and computing of compensation matrices. The procedure has been fully validated with measurements of a 45-element triangular panel array designed for Low Earth Orbit (LEO) satellite tracking that compensates the degradation due to gain and phase imbalances and mutual coupling.
\end{abstract}

Index Terms-Antenna array, calibration, errors, mutual coupling, uncertainty analysis.

\section{INTRODUCTION}

$\mathbf{T}$ HE MOTIVATION of this work comes from the evaluation of new antenna technologies to improve the performance of traditional Earth stations for space communications, regarding the increasing requirement of data upload and download capacity during last decades and launch of new missions [1] [3]. These needs will be faced with new technologies and new architectures for Deep Space missions [4], as well as for Low Earth Orbit (LEO), Medium Earth Orbit (MEO), and Geostationary (GEO) satellites [5], [6].

GEODA is a novel concept of active antenna arrays for LEO satellite tracking in $1.7 \mathrm{GHz}$ [7]. The antenna is formed by 60 triangular panels positioned over a dome with a cylindrical base [Fig. 1(a)]. Each panel is formed by 45 double-stacked patches followed by low noise amplifiers (LNAs) and switchedline phase shifters that control the beamsteering of the array pattern. Panels are organized in 15 identical subarrays (cells) of three elements as shown in Fig. 1(b). GEODA uses adaptive beamforming algorithms based on a spatial reference to track satellites. Therefore, the estimation of an accurate solution of the direction of arrival (DOA) and beamformer performance relies in the calibration process. As the antenna under test (AUT), we have used a triangular panel.

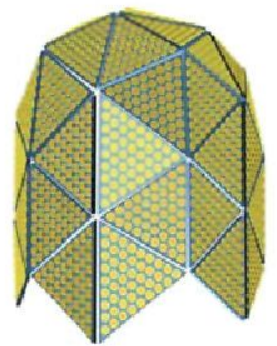

(a)

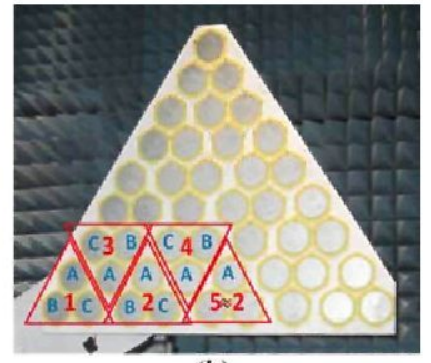

(b)
Fig. 1. GEODA antenna. (a) GEODA. (b) Panel of 45 active elements.

In this work, we faced the demanding calibration process of an active antenna array in terms of the number of measurements and calibration steps. First, the evaluation of manufacturing errors based on the uncertainty analysis was performed. Second, a measurement campaign in a laboratory and an anechoic chamber for the characterization of the antenna was carried out. Finally, the calibration process was defined.

In this letter, we present a new calibration process valid for active antenna arrays with any array geometry. The procedure is valid for any antenna element as errors and mutual coupling are evaluated from the measured radiated field that considers all significant propagating and evanescent modes and coupling between them [8]. Furthermore, we focus on the offline calibration process that is performed in a laboratory and an anechoic chamber before the antenna is installed. The calibration process reduces the number of measurements by geometric fitting based on the antenna array symmetry, while providing an accurate characterization of the antenna array that is used to compensate errors due to gain and phase differences and mutual coupling.

\section{EXPerimental Procedure}

The experimental procedure required for the proposed calibration has four steps:

Step 1) selection of the proper calibration technique based on the uncertainties analysis and mutual coupling evaluation;

Step 2) evaluation of measurement requirements from the calibration technique to define the measurement campaign;

Step 3) procedure for measurement campaigns (automated);

Step 4) post-processing of measurement results for calibration and characterization.

The sequential application of the previous steps is shown in Fig. 2. 


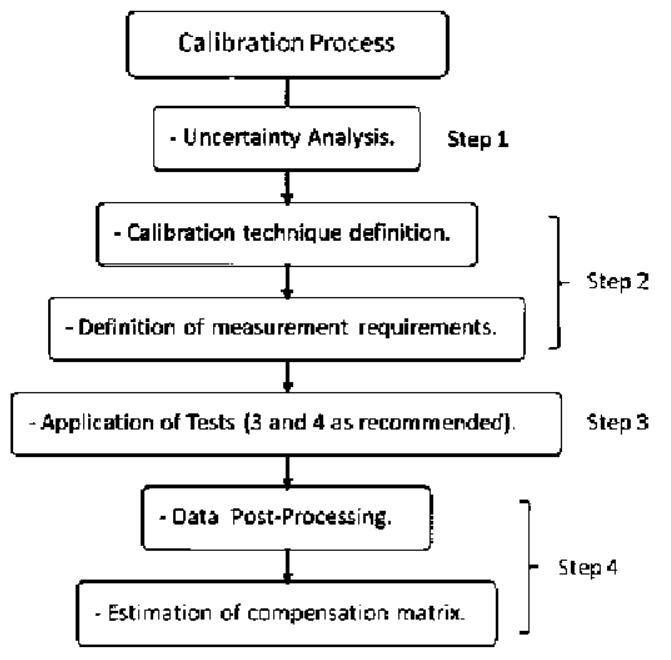

Fig. 2. Diagram of the offline calibration.

TABLE I

SUMMARY OF MEASUREMENTS

\begin{tabular}{|c|c|c|c|}
\hline Test & Output & Faciliny & Equpment \\
\hline I & $\begin{array}{c}\text { Coupling coeficient or the } \\
\text { antenna array }\end{array}$ & \multirow{3}{*}{ Laboratory } & \multirow{3}{*}{$\begin{array}{l}\text { Vector Network } \\
\text { Analyzer. Antentrn } \\
\text { Workstation. RF } \\
\text { Connectors and } \\
\text { waveguides. Power } \\
\text { supply device }\end{array}$} \\
\hline 2 & $\begin{array}{c}S_{21} \text { parameters of branches of } \\
\text { RF circuits. }\end{array}$ & & \\
\hline 3 & $\begin{array}{l}\mathrm{S}_{21} \text { paranneters of brancless of } \\
\mathrm{RF} \text { complete thetwork (No } \\
\text { hybrid couplers jncluded) }\end{array}$ & & \\
\hline 4 & $\begin{array}{c}\text { Active element pattems of sub- } \\
\text { array and complete artay } \\
\text { pattern }\end{array}$ & $\begin{array}{l}\text { Anechoic } \\
\text { Clamber }\end{array}$ & $\begin{array}{c}\text { Anechoic Chamber. } \\
\text { Antemna } \\
\text { Workstation. RF } \\
\text { Connectors and } \\
\text { waveguides. Power } \\
\text { Supply device }\end{array}$ \\
\hline
\end{tabular}

The uncertainty analysis (Step 1) evaluates the effect of errors on the antenna array response based on the signal model for active antenna arrays. As a result, an analytical method for uncertainty evaluation in active antenna arrays was derived [9]. With the analytical method, the impact of amplitude, phase, and antenna element location errors on the array response can be evaluated, and decisions on the selection of components and the most appropriate calibration techniques can be made. In GEODA, we opted for a phase and mutual coupling calibration as these errors had a significant impact on the array performance.

Measurements for characterization, validation, and calibration of the antenna are summarized in Table $I$. The number of subsystems and components in GEODA symbolized a challenge in terms of an exhaustive, complex, and expensive measurements campaign. Tests 1 and 2 are used for characterization and validation of the antenna, while tests 3 and 4 are recommended for calibration.

Finally, post-processing of measurement results in Step 4 derives in the estimation of calibration matrices that will be used to compensate errors.

\section{Proposed Offline Calibration}

Next, the active antenna array pattern model and the expansion of equation for calibration are presented. This section includes the derived expression of the active element pattern and the cost function for compensation of mutual coupling effect, gain, and phase errors.

\section{A. Active Antenna Array Pattern Model}

The active array pattern $Y(\theta, \varphi)$ including errors can be expressed as

$$
Y(\theta, \varphi)=\underline{W^{H}} \underline{M}(\theta, \varphi)
$$

where $\underline{W}$ represents the beamforming vector whose $m$ th term represents the complex beamforming weight for the $m$ th antenna element and $(.)^{H}$ is the Hermitian operator. $W$ can be computed to synthesize an antenna pattern that satisfies an optimization criterion, such as tracking of moving targets or cancellation of interference sources.

$\underline{M}(\theta, \varphi)$ is the array manifold that includes all the error contributions and can be expressed as

$$
\begin{aligned}
& \underline{M}(\theta, \varphi)=\mathrm{C}\left(G_{\mathrm{RF}}+\Delta G_{\mathrm{RF}}\right) \\
& \quad(\underline{G}(\theta, \varphi)+\underline{\Delta G}(\theta, \varphi)) \odot(\underline{A}(\theta, \varphi)+\underline{\Delta A}(\theta, \varphi)) .
\end{aligned}
$$

$M(\theta, \varphi)$ is an $M \times 1$ vector that can be denoted as $\left[\begin{array}{llll}M_{1} & M_{2} & \cdots & M_{M}\end{array}\right]^{T}$, whose $m$ th vector element is a matrix that represents the pattern of the $m$ th antenna element defined in a $(\theta, \varphi)$ grid, and $M$ is the number of elements of the array. $\odot$ represents the Hadamard product. $\mathrm{C}$ is the $M \times M$ mutual coupling matrix. $G_{\mathrm{RF}}, \Delta G_{\mathrm{RF}}$ are $M \times M$ diagonal matrices whose complex elements represent the gain of the $R F$ circuits and the effect of the gain and phase uncertainty sources due to its components. $G(\theta, \varphi)$ is an $M \times 1$ vector of matrices representing the gain and phase of each radiating element in the $(\theta, \varphi)$ grid as $\underline{g}_{m} e^{j \psi_{m}}$ of the $m$ th matrix $M_{m} . \underline{A}$ is an $M \times 1$ vector of matrices representing the ideal steering vector of the array in the $(\theta, \varphi)$ grid as $\underline{A}=\mathrm{e}^{-j(2 \pi / \lambda) \overrightarrow{\mathrm{r}}^{\prime}}$, with $\vec{r}$ being the array location vector, $\hat{r}^{\prime}$ is the unitary direction vector in $(\theta, \varphi), \hat{\mathrm{r}}^{\prime}=\left[\begin{array}{lll}(\sin \theta \cos \varphi & \sin \theta \sin \varphi & \cos \theta\end{array}\right]^{T}$, and $\lambda$ is the wavelength. $\Delta G$ and $\Delta \underline{A}$ are vector of matrices representing the deviation of $G$ and $A$ due to the presence of errors, respectively. Note that in the ideal case with no errors and noise, the expression for the ideal beamformed array pattern $Y_{o}(\theta, \varphi)$ can be written as

$$
\begin{aligned}
Y_{o}(\theta, \varphi) & =\underline{W}_{a}^{H}\left(\mathrm{C} G_{\mathrm{RF}} \underline{G}(\theta, \varphi) \odot \underline{A}(\theta, \varphi)\right) \\
& =\underline{W}_{o}^{H} \underline{M}_{o}(\theta, \varphi) .
\end{aligned}
$$

\section{B. Active Element Pattern Model}

Since the fully excited antenna array can be expressed in terms of the active element pattern and the array factor, the active element pattern is useful in the characterization of an antenna array. Furthermore, the scattering matrix can be computed from active element patterns [10], [11]. The radiated electric field of the $m$ th active element can be written as a function of the radiated electric field $E_{e}$ of each isolated element as

$$
\begin{aligned}
E_{m}= & E_{e}(\theta, \varphi) \cdot\left[e^{-j k_{0}\left(d x_{m} \sin \theta_{0} \cos \varphi_{0}+d y_{m} \sin \theta_{0} \sin \varphi_{0}\right)}\right. \\
& \left.+\sum_{k=1}^{K} S_{k m} e^{-j k_{o}\left(d x_{k} \sin \theta_{0} \cos \varphi_{0}+d y_{k} \sin \theta_{0} \sin \varphi_{0}\right)}\right] \\
& \cdot e^{j k_{o}\left(d x_{m} \sin \theta \cos \varphi+d y_{m} \sin \theta \sin \varphi\right)} .
\end{aligned}
$$




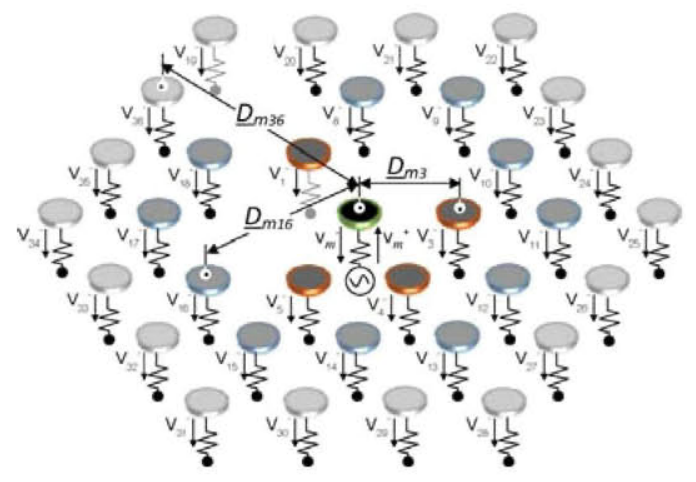

Fig. 3. General planar antenna array model to obtain the active element pattern.

With the active element pattern of the $M$ elements measured for broadside, it is possible to compute the scattering matrix. Equation (5) expresses the active element pattern of the $m$ th element of the array as

$$
E_{m}(\theta, \varphi)=E_{e}(\theta, \varphi) e^{j k_{o}\left(\mu_{n}(m)-\mu_{o}(m)\right)}\left[1+\underline{S}_{m k} \underline{D}_{m k}\right]
$$

where $\underline{S}_{m k}=\left[\begin{array}{llll}S_{m 1} & S_{m 2} & \ldots & S_{m K}\end{array}\right]$ is the $1 \times K$ vector of scattering coefficients of the $m$ th antenna element. $\underline{D}_{m k}$ is the phase differences vector due to the location of the $k$ th neighbor element of the $m$ th array element as depicted in Fig. 3. The $K \times 1$ vector $\underline{D}_{m k}$ for $k=1,2, \ldots, K$ can be expressed as $\left[\begin{array}{llll}e^{-j k_{o}\left(\mu_{o}(1)-\mu_{o}(m)\right)} & e^{-j k_{o}\left(\mu_{o}(2)-\mu_{o}(m)\right)} & \cdots & e^{-j k_{o}\left(\mu_{o}(K)-\mu_{o}(m)\right)}\end{array}\right]^{T}$. $\mu_{o}(m)$ and $\mu_{n}(m)$ represent the phase of the $m$ th element for the broadside and the direction under analysis, respectively. Thus, $e^{j k_{o}\left(\mu_{n}(m)-\mu_{o}(m)\right)}$ can be defined as $R_{p}$ and expressed as follows:

$$
R_{p}=e^{j k_{o}\left(\mu_{n}(m)-\mu_{o}(m)\right)}=e^{j(2 \pi / \lambda) \overrightarrow{\mathrm{r}}_{m} \hat{\mathbf{r}}_{n}^{\prime}} \cdot e^{-j(2 \pi / \lambda) \overrightarrow{\mathrm{r}}_{m} \hat{\mathbf{r}}_{o}^{\prime}} .
$$

Using (6), (5) can be written as

$$
\mathrm{E}_{m}(\theta, \varphi)=\mathrm{E}_{e}(\theta, \varphi) \odot R_{p}\left[1+\underline{S}_{m k} \underline{D}_{m k}\right] .
$$

Now, the term $\mathrm{E}_{i}$ can be introduced as the isolated element pattern considering its location in the array expressed by $\mathrm{E}_{i}=$ $\mathrm{E}_{e}(\theta, \varphi) \odot R_{p}$ simplifying (7). Furthermore, for $\left(\theta_{0}, \varphi_{0}\right)$ where $\underline{S}_{m k} \underline{D}_{m k}$ can be directly estimated, the active element pattern can be expressed as

$$
\mathrm{E}_{m}\left(\theta_{0}, \varphi_{0}\right)=\mathrm{E}_{i}\left(\theta_{0}, \varphi_{0}\right)\left[1+\underline{S}_{m k} \underline{D}_{m k}\right] .
$$

\section{Estimation of Compensation Matrices}

The theory for the estimation of a complete compensation matrix including mutual coupling effect, phase, and gain error is addressed in this section. Regarding (2), a new term for a general approximation of errors can be included and expressed as

$$
M_{e}(\theta, \varphi)=\mathrm{C}\left(\Delta G_{\mathrm{RF}} \Delta G(\theta, \varphi) \Delta A(\theta, \varphi)\right)
$$

where $M_{e}(\theta, \varphi)$ is the general matrix of errors and mutual coupling of the array manifold. $\Delta G(\theta, \varphi), \Delta A(\theta, \varphi)$ are $M \times M$ diagonal matrices for (9). Coefficients of $M_{e}(\theta, \varphi)$ include amplitude and phase contribution of uncertainties and coupling coefficients. When active element patterns are measured with the
TABLE II

STANDARD DEVIATION OF TEST 2 Results

\begin{tabular}{c|c|c|c|c|c|c}
\hline${\text { Phase shifter }\left[{ }^{\circ}\right]}^{\circ}$ & 0 & 60 & 120 & 180 & 240 & 300 \\
\hline$\sigma\left(\Psi_{\mathrm{RE}}\right)$ & 5.20 & 5.10 & 4.74 & 5.18 & 5.10 & 5.23 \\
\hline$\sigma\left(\mathrm{G}_{\mathrm{RE}}\right)$ & 0.23 & 0.26 & 0.24 & 0.21 & 0.26 & 0.26 \\
\hline
\end{tabular}

RF circuit assembled, errors due to dissimilar branches of RF circuits can be included in $M_{e}(\theta, \varphi)$. Regarding this, the array manifold can be written as

$$
\underline{M}(\theta, \varphi)=M_{e}(\theta, \varphi) G_{\mathrm{RF}}(\underline{G}(\theta, \varphi) \odot \underline{A}(\theta, \varphi)) .
$$

To estimate the matrix $M_{e}(\theta, \varphi)$, it is possible to evaluate the (11) using a Least Square matrix inversion

$$
\min _{M_{e}(\theta, \varphi)}\left\|\underline{M}(\theta, \varphi)^{T}-\left[G_{R F}(\underline{G}(\theta, \varphi) \odot \underline{A}(\theta, \varphi))\right]^{T} M_{e}(\theta, \varphi)\right\|_{2}^{2} .
$$

The Least Squares solution is the matrix $M_{e}(\theta, \varphi)$, which includes the unknown vector of coefficients. The normal system of equations using the transpose of each vector of (11) is given by

$$
\begin{aligned}
\left(G_{\mathrm{RF}}(\underline{G}(\theta, \varphi) \odot \underline{A}(\theta, \varphi))\right] \cdot \\
{\left.\left[G_{\mathrm{RF}}(\underline{G}(\theta, \varphi) \odot \underline{A}(\theta, \varphi))\right]^{T}\right) M_{e}(\theta, \varphi) } \\
\quad=\left[G_{\mathrm{RF}}(\underline{G}(\theta, \varphi) \odot \underline{A}(\theta, \varphi))\right] \underline{M}(\theta, \varphi)^{T} .
\end{aligned}
$$

Finally, the solution for $M_{e}(\theta, \varphi)$ can be expressed as

$$
\begin{aligned}
M_{e}(\theta, \varphi)=\left(\begin{array}{l}
\left.\left[G_{\mathrm{RF}}(\underline{G}(\theta, \varphi) \odot \underline{A}(\theta, \varphi))\right] \cdot\right)^{-1} \\
{\left[G_{\mathrm{RF}}(\underline{G}(\theta, \varphi) \odot \underline{A}(\theta, \varphi))\right]^{T}}
\end{array}\right. \\
\cdot\left[G_{\mathrm{RF}}(\underline{G}(\theta, \varphi) \odot \underline{A}(\theta, \varphi))\right] \underline{M}(\theta, \varphi)^{T} .
\end{aligned}
$$

\section{EXPERIMENTAL RESULTS}

Laboratory and anechoic chamber measurements have been performed to complete the measurement procedure. Starting with the test 2 , it was necessary to carry out 4860 measurements of the $S_{21}$ parameter to evaluate all the combinations of the six states of phase-shifters and three states of LNA of each patch in a triangular panel. Results in Table II show the standard deviation of the phase $\Psi_{\mathrm{RF}}$ and gain $G_{\mathrm{RF}}$ of the RF circuits.

Fig. 4 depicts different $\varphi$-cuts of the normalized power pattern of the triangular panel pointing to $\theta=30^{\circ}$ and $\varphi=0^{\circ}$ at $1.7 \mathrm{GHz}$ from test 4 . The active array has a mispointing loss of $1.5 \mathrm{~dB}$ due to the pointing error of $5^{\circ}$, thus leading to a reduction in the achievable bit rate or maximum range in the link budget of the communication system.

Arrays with nonuniform distributed elements improve the performance of the array in direction of arrival problem when periodic nulls due to coupling effect. Those periodic nulls make patterns of uniform distributed antenna arrays blind to that specific direction of arrival [11], while that effect is quite reduced with nonuniform distributed antenna arrays [12]. Regarding the discussion above, since the AUT has nonuniform distributed elements and phase shift between feeding ports of elements, an enhanced performance in terms of blind affect due to mutual coupling was obtained, but the front phase required compensation in order to reduce the mispointing error of the antenna. Moreover, the proposed algorithm for calibration has 


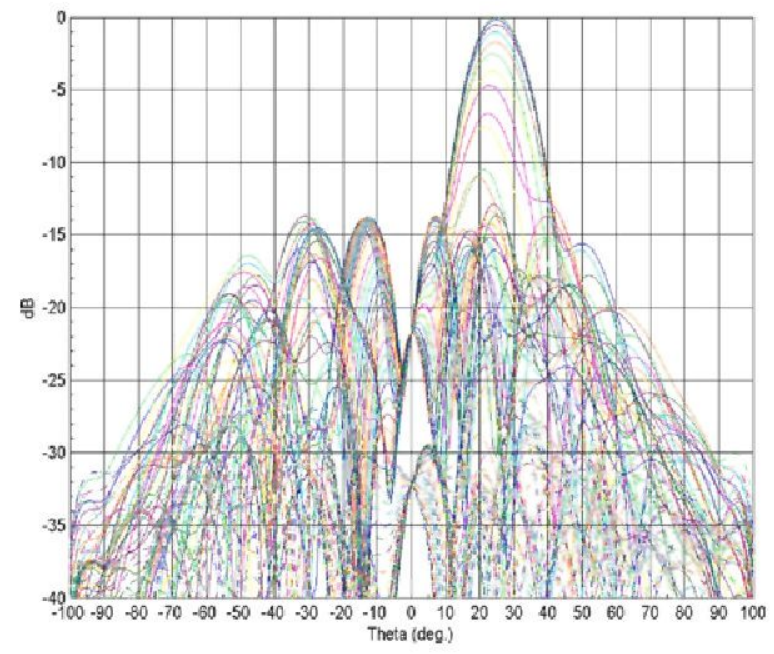

Fig. 4. Anechoic chamber measurement of the pattern of the triangular active array panel at $\theta=30^{\circ}, \varphi=0^{\circ}$, and $f=1.7 \mathrm{GHz}$.

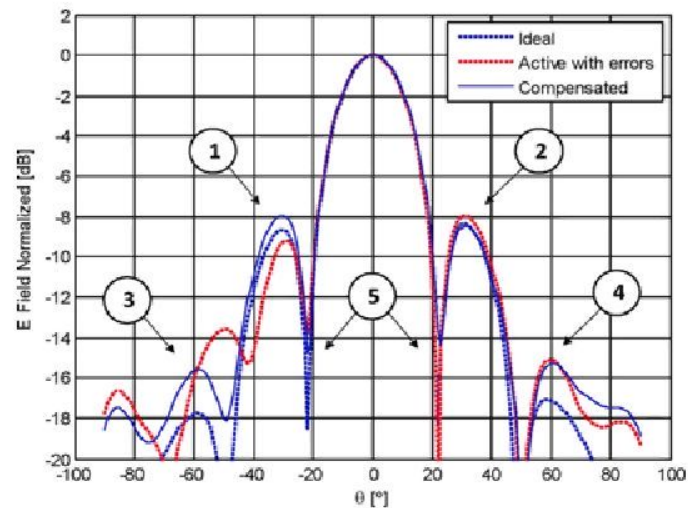

(a)

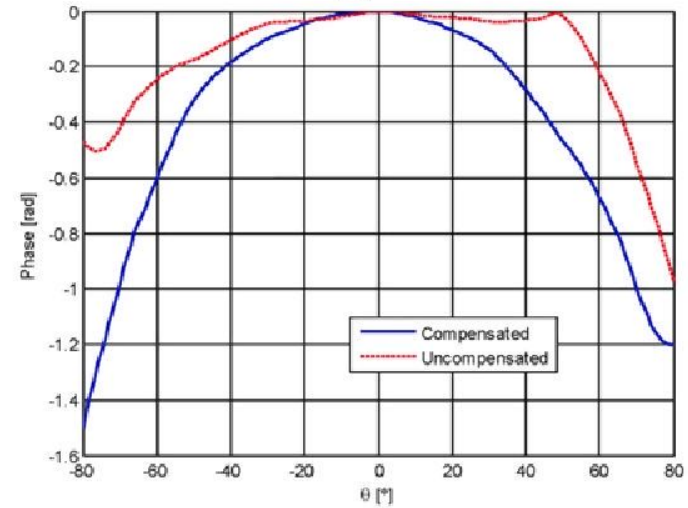

(b)

Fig. 5. Compensated results of the AUT based on the $M_{\mathrm{e}}(\theta, \varphi)$ matrix. (a) E-field of the AUT. (b) Phase of the Cell 4 of the AUT.

an excellent performance compensating errors as shown in amplitude and phase depicted in Fig. 5.

Additionally, using measurements of the subarray of cells 1-4 in Figs. 1, Fig. 5(a) shows key points where compensated pattern presents about $-8 \mathrm{~dB}$ of sidelobe in points 1 and 2 . Regarding sidelobes in points 3 and 4, the compensated pattern has a considerable reduction of about $2 \mathrm{~dB}$ with respect to the active pattern with errors. As a conclusion, the compensated pattern is more symmetrical than the active pattern (before compensation) and presents a good performance in terms of the com- plete pattern. Moreover, the compensation of the phase front is depicted in Fig. 5(b), which presents an important reduction of errors enhancing the symmetry of the phase and reducing the ripple effect due to the presence of mutual coupling. Measurements of the active element pattern were done fitting the geometrical center of cells to the spherical system of the anechoic chamber.

The error compensation shown for Cell 4 in Fig. 5(b) of about $0.2 \mathrm{rad}$ has an impact on the AUT of about $4 \mathrm{~dB}$ of reduction of the interference signals at $\theta=-50^{\circ}$ and a gain improvement at $\theta=-30^{\circ}$ as presented in Fig. 5(a).

\section{CONCLUSION}

In this letter, a novel offline calibration procedure for active antenna arrays has been presented. The proposed procedure is valid for any array geometry and antenna element being capable of compensating phase, gain, and mutual coupling effects. Moreover, it is possible to complete the calibration with a significant reduction of measurements and cost. The proposed procedure for calibration process is presented from a system-level framework, including the measurements schedule as an essential part in the process of calibration and characterization of the array.

The procedure has been applied and validated using a triangular panel of 45 active patches used for tracking LEO satellites. Experimental results have shown a reduction of about $73 \%$ in time and cost while compensating the effect of errors and mutual coupling.

\section{REFERENCES}

[1] F. Davarian, "Uplink arrays for the deep space network," Proc. IEEE, vol. 95, no. 10, pp. 1923-1930, Oct. 2007.

[2] B. J. Geldzahler, J. J. Rush, and L. J. Deutsch, "Engineering the next generation deep space network," in Proc. IEEE MTT-S Int. Microw. Symp., Honolulu, HI, Jun. 2007, pp. 931-934.

[3] F. Amoozegar, L. Paal, A. Mileant, and D. Lee, "Analysis of errors for uplink array of 34-m antennas for deep space applications," in Proc. IEEE Aerosp. Conf., Big Sky, MT, Mar. 2005, pp. 1235-1257.

[4] J. C. I. o. Technology, "Deep space network brochure," Mar. 2010 [Online]. Available: http://deepspace.jpl.nasa.gov/dsn/brochure/

[5] R. Martinez and M. A. Salas Natera, "On the use of ground antenna arrays for satellite tracking: Architecture, beamforming, calibration and measurements," in Proc. 61st Int. Astronaut. Congress, Prague, Czech Republic, 2010, pp. 1-7.

[6] M. A. Salas Natera, A. García Aguilar, J. Mora Cuevas, J. M. Fernández, P. Padilla de la Torre, J. García-Gasco Trujillo, R. Martínez Rodriguez-Osorio, M. Sierra-Pérez, L. De Haro Ariet, and M. Sierra Castafier, "New antenna array architectures for satellite communications," in Advances in Satellite Communications. Rijeka, Croatia: InTech, 2011, ch. 7, pp. 167-194.

[7] M. S. Pérez, A. Torre, J. L. Masa Campos, D. Ktorza, and I. Montesinos, "GEODA: Adaptive antenna array for metop satellite signal reception," in Proc. 4th ESA Int. Workshop Tracking, Telemetry Command Syst. Space Appl., Darmstadt, Germany, Sep. 2007, pp. 1-4.

[8] P. Clarricoats, "Effects of mutual coupling in conical horn arrays," Inst. Elect. Eng. Proc. H Microw., Opt. Antennas, vol. 131, no. 3, pp. 165-171, Jun. 1984.

[9] M. Salas Natera and R. Martínez, "Analytical evaluation of uncertainty on active antenna arrays," IEEE Trans. Aerosp. Electron.Syst., vol. 48, no. 3, pp. 1903-1913, Jul. 2011.

[10] D. M. Pozar, "A relation between the active input impedance and the active element pattern of a phased array," IEEE Trans. Antennas Propag., vol. 51, no. 9, pp. 2486-2489, Sep. 2003.

[11] D. M. Pozar, "The active element pattern," IEEE Trans. Antennas Propag., vol. 42, no. 8, pp. 1176-1178, Aug. 1994.

[12] D. Segovia-Vargas, R. Martin-Cuerdo, and M. Sierra Pérez, "Mutual coupling effects correction in microstrip arrays for direction-of-arrival (DOA) estimation," Inst. Elect. Eng. Proc. Microw., Antennas Propag., vol. 149, no. 2, pp. 113-118, Apr. 2002. 